\title{
DYNAMIC DAMAGE AND FAILURE IN VISCO-PLASTIC MATERIALS
}

\author{
ZHENG JIAN \\ Laboratory for Non-linear Mechanics of Continuous Media, Institute of Mechanics, \\ Chinese Academy of Science, Beijing 100080, People's Republic of China \\ and \\ WANG ZE-PING \\ Department of Mechanical and Production Engineering, National University of Singapore, \\ 10 Kent Ridge Crescent, 0511, Singapore
}

\begin{abstract}
In this paper, a dynamic damage model in ductile solids under the application of a dynamic mean tensile stress is developed. The proposed model considers void nucleation and growth as parts of the damage process under intense dynamic loading (strain rates $\epsilon \geqslant 10^{3} \mathrm{~s}^{-1}$ ). The evolution equation of the ductile void has the closed form, in which work-hardening behavior, rate-dependent contribution and inertial effects are taken into account. Meanwhile, a plate impact test is performed for simulating the dynamic fracture process in LY12 aluminum alloy. The damage model is incorporated in a hydrodynamic computer code, to simulate the first few stress reverberations in the target as it spalls and postimpact porosity in the specimen. Fair agreement between computed and experimental results is obtained. Numerical analysis shows that the influence of inertial resistance on the initial void growth in the case of high loading rate can not be neglected. It is also indicated that the dynamic growth of voids is highly sensitive to the strain rates.
\end{abstract}

\section{INTRODUCTION}

DYNAMIC DUCTILE fracture such as spallation, tensile failure of smooth or notched bar specimens, and adiabatic shear bands is concerned with the nucleation, growth and coalescence of microvoids in ductile solids. Under the dynamic loading, especially the intense dynamic loading (strain rate $\dot{\epsilon} \geqslant 10^{3} \mathrm{~s}^{-1}$ ), the behavior of damage and fracture in ductile materials is quite different from that of static or quasi-static loading. The major factors of influence on the process of dynamic damage are strain rate, inertial effects and thermal effect from plastic deformation work. Experimental observations show that the problem of dynamic frature is more complex than that of static fracture. Much attention has been given to static fracture; the dynamic problem has remained relatively unexplored. Meyers and Aimone [1] pointed out that the rate dependence, inertial effects and effects of temperature from local plastic deformations in the materials greatly influenced the process of dynamic damage and fracture in solids under intense dynamic loading.

Grady [2] studied local inertial effects in dynamic fragmentation. He pointed out that the inertial effects played an important role in the dynamic fracture behavior of materials. After studying the inertial effects in a tensile test at a high rate of loading in detail, Regazzoni et al. [3] concluded that at high strain rates $\left(\dot{\epsilon} \geqslant 10^{3} \mathrm{~s}^{-1}\right)$ inertia and strain rate sensitivity led to effects of the same order of magnitude. Tvergaard and Needleman [4] obtained the same result that inertial effects were an important factor during the growth of voids in porous ductile materials under very high strain rates. Johnson [5] discussed this problem qualitatively, but the inertial effects were neglected in his ductile void growth model.

In this paper, the mathematical model of dynamic ductile failure under the application of dynamic mean stress is developed. The effects of material rate dependence, inertial effects and work hardening are investigated in detail. To simplify the theoretical analysis, some assumptions are employed that the matrix material is incompressible and the spherical geometry is maintained during the growth of voids. In addition, when dealing with void growth, the initial elastic and elastic-plastic phases of the process are neglected and it is assumed that all of the matrix material is in the plastic state. Although this assumption is not always reasonable, it is at least suitable for cases where the 
plastic response dominates. These assumptions afford a great simplification in the theoretical derivation.

As an application of the model of dynamic ductile failure proposed in this work, the process of spallation in LY12 aluminum alloy is simulated. Comparison of calculations and experimental results is reasonbly good.

\section{A MODEL OF DYNAMIC DUCTILE FRACTURE}

Nucleation and growth of voids are included in the failure model presented in this work. Define a damage variable, $\Phi$, called porosity as $\Phi=V_{\mathrm{h}} / V$, where $V_{\mathrm{h}}$ denotes the volume of the void, $V$ denotes the volume of the solid plus $V_{\mathrm{h}}$. The fracture criterion of the material is $\Phi \geqslant \Phi_{\text {crit }}$, where $\Phi_{\text {crit }}$ is the critical porosity of fracture. We suppose that the porosity rate $\Phi$ is composed of two parts

$$
\dot{\Phi}=\dot{\Phi}_{\mathrm{n}}+\dot{\Phi}_{\mathrm{g}},
$$

where "•" denotes differential of time $t, \dot{\Phi}_{\mathrm{n}}$ is the contribution from the nucleation of voids, $\dot{\Phi}_{\mathrm{g}}$ is the contribution from the growth of existing voids.

\subsection{Nucleation of void}

Curran et al. [6] pointed out that because of the variety and complexity of the specific nucleation mechanisms, it was suggested that a priori quantitative derivations of analytical models of microflaw nucleation in structures are an unrealistic goal. They considered that the major factors to influence microflaw nucleation were stress, strain and temperature. Chu and Needleman [7] gave a description of void nucleation that followed a normal distribution about some mean stress. This idealization is employed in the present study, $\dot{\Phi}_{\mathrm{n}}$ is given by [7]

$$
\begin{aligned}
& \dot{\Phi}_{\mathrm{n}}=A\left(\dot{\sigma}_{\mathrm{m}}+\frac{1}{3} \dot{\sigma}_{\mathrm{kk}}\right)+B \dot{\epsilon}^{\mathrm{p}} \\
& A= \begin{cases}\frac{f_{\mathrm{n}}}{s_{\mathrm{n}} \sqrt{2 \pi}} \exp \left[-\frac{1}{2}\left(\frac{\sigma_{\mathrm{m}}+\frac{1}{3} \sigma_{\mathrm{kk}}-\sigma_{\mathrm{n}}}{s_{\mathrm{n}}}\right)^{2}\right], & \sigma_{\mathrm{m}}+\frac{1}{3} \sigma_{\mathrm{kk}}-\sigma_{\mathrm{n}}>0 \\
0, & \sigma_{\mathrm{m}}+\frac{1}{3} \sigma_{\mathrm{kk}}-\sigma_{\mathrm{n}} \leq 0\end{cases}
\end{aligned}
$$

where $\sigma_{\mathrm{m}}$ is the microscopic effective stress in the matrix material, $\frac{1}{3} \sigma_{\mathrm{kk}}$ is the macroscopic mean stress and $\epsilon^{\mathrm{p}}$ is the current value of the effective plastic strain rate which represents the actual microscopic strain state in the matrix material. To describe a spallation type failure, we neglected the nucleation rate due to plastic strain, namely, $B=0 . f_{\mathrm{a}}$ is the volume fraction of void nucleating particles, $s_{\mathrm{n}}$ is the corresponding standard deviation and $\sigma_{\mathrm{n}}$ is the mean nucleation threshold stress.

\subsection{Growth of void}

We assume that the porous ductile material is statistically homogeneous and isotropic so that it can be effectively modeled by a homogeneous isotropic solid material. Moreover, we assume that the volumetric behavior of this effective homogeneous material is described by the PVE $\Phi$ relation and a pore-collapse relation that depends on the properties of the matrix material. In addition, we assume that the spherical geometry of the void is maintained during the growth of a void.

With numerical analysis Carroll and Holt [8] remarked that the elastic and elastic-plastic phases of a void growth are small compared with the plastic phase during the void growth. When dealing with the void growth in materials, we can neglect the initial elastic and elastic-plastic phases of the process and go immediately to the case of fully plastic deformation around the void. The assumptions of matrix incompressibility during pore collapse are adopted, which leads to great simplification of a thorough analysis of the pore collapse of our spherical model.

A simplified model of the porous element is assumed. Consider a single spherical void of radius $a$ in a sphere of radius $b$ subject to external stress $P(t)$ (Fig. 1). A damage variable, the distention $\alpha$, is introduced for the description of the damage of fracture in the ductile materials. $\alpha$ is defined as

$$
\alpha=\frac{b^{3}}{b^{3}-a^{3}} .
$$




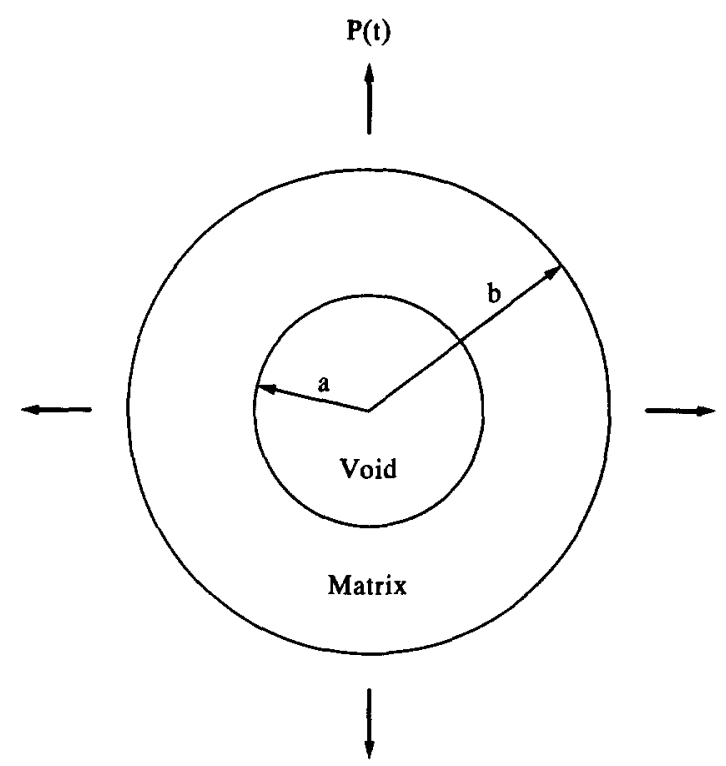

Fig. 1. Porous ductile material model.

Consider the matrix material and void to be a system, work done by the applied external pressure $P(t)$ is equal to the change of the system energy, namely,

$$
\Delta E_{\mathrm{K}}+\Delta E_{\mathrm{s}}+\Delta E_{\mathrm{i}}=W
$$

where $\Delta E_{\mathrm{K}}, \Delta E_{\mathrm{S}}$ and $\Delta E_{\mathrm{i}}$ denote the changes of the kinetic energy, the surface energy of the voids and the internal energy in the system, respectively. $W$ denotes the work done by the applied external pressure $P(t)$.

As mentioned in the previous section, we neglect the initial elastic and elastic-plastic phases of the process and begin to consider fully plastic deformation in the solid around the void.

Johnson's results [5] are used in this work,

$$
\begin{gathered}
r^{3}=r_{0}^{3}-B(t), \quad B(t)=a_{0}^{3} \frac{\alpha_{0}-\alpha}{\alpha_{0}-1} \\
\frac{B(t)}{a^{3}}=\frac{\alpha_{0}-\alpha}{\alpha-1}, \quad \frac{B(t)}{b^{3}}=\frac{\alpha_{0}-\alpha}{\alpha} \\
\dot{r}=\frac{\dot{B}(t)}{3 r^{2}},
\end{gathered}
$$

where "•" denotes the differential with respect to time $t . r$ is the Lagrangian radial position coordinate in the solid surrounding the void and $a_{0}$ is the initial radius of the void.

Consider $\Delta E_{\mathrm{K}}, \Delta E_{\mathrm{s}}, \Delta E_{\mathrm{i}}$ and $W$, respectively. $\Delta E_{\mathrm{K}}$ is given by

$$
\Delta E_{\mathrm{K}}=E_{\mathrm{K}}(\alpha)-E_{\mathrm{K}}\left(\alpha_{0}\right),
$$

where

$$
E_{\mathrm{K}}(\alpha)=\int_{n}^{b} \frac{1}{2} \dot{r}^{2} \rho_{\mathrm{S}} 4 \pi r^{2} \mathrm{~d} r
$$

$\rho_{\mathrm{s}}$ is the density of the matrix material.

Using eqs (5)-(7), we have

$$
E_{\mathrm{K}}(\alpha)=\left[\frac{4 \pi a_{0}^{3}}{9\left(\alpha_{0}-1\right)}\right] \frac{a_{0}^{2} \rho_{\mathrm{s}}}{2\left(\alpha_{0}-1\right)}\left(\frac{\alpha_{0}-1}{\alpha-1}\right)^{1 / 3}\left[1-\left(\frac{\alpha-1}{\alpha}\right)^{1 / 3}\right] \dot{\alpha}^{2} .
$$


We can also obtain the expression for $\Delta E_{\mathrm{s}}$

$$
\Delta E_{\mathrm{S}}=E_{\mathrm{S}}(\alpha)-E_{\mathrm{S}}\left(\alpha_{0}\right)
$$

where

$$
E_{\mathrm{S}}(\alpha)=\left[\frac{4 \pi a_{0}^{3}}{9\left(\alpha_{0}-1\right)}\right] \frac{9\left(\alpha_{0}-1\right)^{1 / 3} \gamma}{a_{0}}(\alpha-1)^{2 / 3}
$$

$\gamma$ is the surface energy expended per unit area during the hole expanding.

The problem studied in the present paper is mainly concerned with the dynamic growth of a spherical void under the intense dynamic loading. The process of the dynamic growth of a ductile void can be approximately considered as an adiabatic process. In addition, we suppose that there is no heat source in the system. With these assumptions, the change of the specific internal energy is given by

$$
\mathrm{d} e_{\mathrm{i}}=\frac{1}{\rho} S_{j k} \mathrm{~d} \epsilon_{j k}^{\mathrm{p}}=\frac{1}{\rho} \sigma_{\mathrm{e}} \mathrm{d} \epsilon^{\mathrm{p}},
$$

where $S_{j k}$ are deviatoric stress tensor components, $\epsilon_{j k}^{\mathrm{p}}$ are plastic strain tensor components, $\sigma_{\mathrm{e}}$ and $\epsilon^{\mathrm{p}}$ denote the effective stress and effective plastic strain in the matrix material, respectively.

The matrix material is assumed to be rate sensitive, linear work hardening and visco-plastic. The constitutive relation is supposed to be

$$
\sigma_{\mathrm{e}}=Y_{0}+H \epsilon^{\mathrm{p}}+\eta \dot{\epsilon}^{\mathrm{p}},
$$

where $Y_{0}$ is the yield stress of the matrix material, $H$ is a linear work-hardening coefficient, $\eta$ is the material viscosity. $\dot{\epsilon}^{\mathrm{p}}$ is the effective plastic strain rate. Since we assume a plastic deformation process with spherical symmetry, the equivalent plastic strain $\epsilon^{\mathrm{p}}$ is given by Johnson and Meller [9]

$$
\epsilon^{\mathrm{p}}=2 \ln \frac{r}{r_{0}} .
$$

The change of internal energy in the system is

$$
\Delta E_{\mathrm{i}}=\frac{1}{\rho} \int_{a}^{b}\left[\int_{0}^{C \rho} \sigma_{\mathrm{e}}(\epsilon) \mathrm{d} \epsilon\right] 4 \pi \rho r^{2} \mathrm{~d} r .
$$

By means of eqs (5)-(7) and (14), we finally have

$$
\Delta E_{\mathrm{i}}=\left[\frac{4 \pi a_{0}^{3}}{9\left(\alpha_{0}-1\right)}\right]\left[F_{3}(\alpha)+F_{4}(\alpha)+F_{5}(\alpha) \dot{\alpha}\right],
$$

where

$$
\begin{gathered}
F_{3}(\alpha)=2 Y_{0}\left(\ln \frac{\alpha-1}{\alpha_{0}-1}+\alpha \ln \frac{\alpha}{\alpha-1}-\alpha_{0} \ln \frac{\alpha_{0}}{\alpha_{0}-1}\right) \\
F_{4}(\alpha)=\frac{4}{3} H\left[\left(\alpha-\alpha_{0}\right) F(\alpha)+\frac{\alpha_{0}}{2}\left(\ln \frac{\alpha_{0}}{\alpha}\right)^{2}-\frac{\alpha_{0}-1}{2}\left(\ln \frac{\alpha_{0}-1}{\alpha-1}\right)^{2}\right] \\
F(\alpha)=\int_{h_{0}}^{h_{1}} \frac{\ln (h+1)}{h} \mathrm{~d} h \\
h_{0}=\frac{\alpha_{0}-\alpha}{\alpha-1}, \quad h_{1}=\frac{\alpha_{0}-\alpha}{\alpha}
\end{gathered}
$$




$$
F_{5}(\alpha)=\frac{2}{3} \eta\left(\ln \frac{\alpha-1}{\alpha}+\ln \frac{\alpha_{0}}{\alpha_{0}-1}\right) .
$$

Functions $F_{3}(\alpha), F_{4}(\alpha)$ and $F_{5}(\alpha)$ denote the influence of the yield stress of the matrix material, the linear work hardening and material viscosity on the increment of internal energy, respectively. The change of work done by the applied external pressure $P(t)$ is given by

$$
\mathrm{d} W=-4 \pi b^{2} P(t) \mathrm{d} b
$$

where $P(t)$ is negative in tensile and positive in compaction.

From eqs (5) and (6), the following expression can be obtained

$$
W=-\frac{4 \pi}{3} \frac{a_{0}^{3}}{\alpha_{0}-1} \int_{\alpha_{0}}^{\alpha} P(t) \mathrm{d} \alpha .
$$

The applied external pressure $P(t)$ can be considered as a function of distention $\alpha$ by means of the equation of state in the solid surrounding the void, namely [10],

$$
P(t)=P[\alpha(t)]
$$

In terms of eq. (24), eq. (23) becomes

$$
W=-\frac{4 \pi}{3} \frac{a_{0}^{3}}{\alpha_{0}-1} \int_{\alpha_{0}}^{\alpha} P(\alpha) \mathrm{d} \alpha .
$$

This expression is conveniently used in numerical simulation of dynamic ductile damage and fracture in solids. Substitution of eqs (8)-(12), (17) and (23) into eq. (4) gives

$$
F_{1}(\alpha) \dot{\alpha}^{2}+F_{5}(\alpha) \dot{\alpha}+F_{7}(\alpha)=0,
$$

where

$$
\begin{gathered}
F_{1}(\alpha)=\frac{\rho a_{0}^{2}}{2\left(\alpha_{0}-1\right)}\left(\frac{\alpha_{0}-1}{\alpha-1}\right)^{1 / 3}\left[1-\left(\frac{\alpha-1}{\alpha}\right)^{1 / 3}\right] \\
F_{7}(\alpha)=F_{2}(\alpha)+F_{3}(\alpha)+F_{4}(\alpha)+3 \int_{\alpha_{0}}^{\alpha} P(\beta) \mathrm{d} \beta-F_{1}\left(\alpha_{0}\right) \dot{\alpha}_{0}^{2}-F_{2}\left(\alpha_{0}\right) \\
F_{2}(\alpha)=\frac{9\left(\alpha_{0}-1\right)^{1 / 3} \gamma}{a_{0}}(\alpha-1)^{2 / 3} .
\end{gathered}
$$

Equation (26) is the relationship from which we obtain the rate-dependent response of the void growth under dynamic loading. The terms in eq. (26) have a clear physical significance. The first term $F_{1}(\alpha) \dot{\alpha}^{2}-F_{1}\left(\alpha_{0}\right) \dot{\alpha}_{0}^{2}$ on the left-hand-side of eq. (26) represents inertial resistance to the void growth. The second term $F_{5}(\alpha) \dot{\alpha}$ denotes the influence of the material viscosity on the void growth. The third term $F_{7}(\alpha)$ is the sum of the total effects of the applied external pressure, the change of the surface energy of the voids, work hardening and yield stress in the solid surrounding the void on the void growth. These effects can also be isolated and studied in great depth. From eq. (26), the void growth rates $\dot{\alpha}$ can be given by

$$
\dot{\alpha}=\frac{1}{2 F_{1}(\alpha)}\left\{-F_{5}(\alpha)+\sqrt{\left[F_{5}(\alpha)\right]^{2}-4 F_{1}(\alpha) F_{7}(\alpha)}\right\} .
$$

If the inertial effects are neglected, from eq. $26, \dot{\alpha}$ is reduced to

$$
\dot{\alpha}=-\left[F_{7}(\alpha)-F_{1}\left(\alpha_{0}\right) \dot{\alpha}_{0}^{2}\right] / F_{5}(\alpha) .
$$

When the void grows, namely, $\dot{\alpha} \geqslant 0$, the following inequality must be satisfied

$$
P(\alpha) \leqslant-\frac{1}{3}\left[\frac{\mathrm{d} F_{2}(\alpha)}{\mathrm{d} \alpha}+\frac{\mathrm{d} F_{3}(\alpha)}{\mathrm{d} \alpha}+\frac{\mathrm{d} F_{4}(\alpha)}{\mathrm{d} \alpha}\right] .
$$


With the help of eqs (18), (19) and (29), inequality (32) becomes

$$
P(\alpha) \leqslant-\frac{1}{3}\left[\frac{6 \gamma}{a_{0}}\left(\frac{\alpha_{0}-1}{\alpha-1}\right)^{1 / 3}+\frac{4 H}{3} F(\alpha)-\frac{8 H}{3} \frac{\alpha_{0}-1}{\alpha-1} \ln \frac{\alpha-1}{\alpha_{0}-1}+2 Y_{0} \ln \frac{\alpha}{\alpha-1}\right] .
$$

Let

$$
P_{\text {crit }}(\alpha)=-\frac{1}{3}\left[\frac{6 \gamma}{a_{0}}\left(\frac{\alpha_{0}-1}{\alpha-1}\right)^{1 / 3}+\frac{4 H}{3} F(\alpha)-\frac{8 H}{3} \frac{\alpha_{0}-1}{\alpha-1} \ln \frac{\alpha-1}{\alpha_{0}-1}+2 Y_{0} \ln \frac{\alpha}{\alpha-1}\right]
$$

where $P_{\text {crit }}(\alpha)$ is the threshold stress for the dynamic growth of voids. The critical condition that the applied external pressures $P(\alpha)$ must satisfy for the void growth is

$$
P(\alpha)<P_{\text {crit }}(\alpha)
$$

The threshold stress $P_{\text {crit }}(\alpha)$ is associated with the distention and material parameters. Carroll and Holt [8] obtained a similar result, but they did not consider the influence of the change of the surface energy of the voids and material work hardening.

The condition of the threshold mean stress [eq. (35)] implies an interesting fact that the threshold stress for the void dynamic expanding is not associated with the loading rates, only with the distention $\alpha$ of the porous ductile material as well as the material parameters, i.e. for the condition of either dynamic or quasi-static loading, the threshold stresses for the void growth are the same.

\subsection{Numerical analysis}

To investigate the effects of inertia, the material viscosity, under the different rate loading, we numerically analyse the analytical eqs (30) and (31) previously developed. In order to simplify the analysis, the material is assumed to be subjected to a linearly increasing hydrostatic pressure

$$
P(\alpha)=P_{0}+G \alpha,
$$

where $P_{0}=P_{\text {crit }}\left(\alpha_{0}\right)$ and $G$ are constants.

In this section, pure copper is selected to be the material for the numerical analysis. The major material parameters used for calculations are listed in Table 1.

Theoretical analysis and experimental observations $[5,6,11,12]$ have evidenced that the material viscosity (rate-dependent sensitivity), especially in the high strain-rate range, has a great influence on the process of damage and fracture in solids. The mathematical model of dynamic growth of a void presented in the present work also shows the same result. In general, the material viscosity $\eta$ is considered to be proportionate to $1 / \sqrt{\dot{\epsilon}}$, namely $[11,13]$

$$
\eta \sim \frac{1}{\sqrt{\dot{\epsilon}}}
$$

This means that the higher the strain rate in the material is, the lower the material viscosity is. We may estimate that for $\dot{\epsilon} \sim 10^{3} \mathrm{~s}^{-1}, \eta \sim 1.0(\mathrm{GPa} \cdot \mu \mathrm{s})$ and for $\dot{\epsilon} \sim 10^{5} \mathrm{~s}^{-1}, \eta \sim 0.1$ (GPa $\left.\mu \mathrm{s}\right)$. In Fig. 2, two sets of curves are given in terms of eq. (30). Profiles show the changes of distention rate $\dot{\alpha}$ along with $\alpha$ for different viscosity values $\eta$ under the different rates of the applied external pressure. Figure 2 is for $G=0.3,0.1$ (equal approximately to the loading rates of $10.0 \mathrm{GPa} / \mu \mathrm{s}$ and $1.0 \mathrm{GPa} / \mu \mathrm{s}$ ) respectively. It is obvious that the effects of different viscosity $\eta$ on the change of $\dot{\alpha}$ along with $\alpha$ is great. The change becomes larger as the loading rates increase. These important results indicate that

Table 1. Material parameters for pure copper-like material

\begin{tabular}{lcccc}
\hline$\rho\left(\mathrm{g} / \mathrm{cm}^{3}\right)$ & $\gamma\left(\mathrm{J} / \mathrm{m}^{2}\right)$ & $Y_{0}(\mathrm{GPa})$ & $H(\mathrm{GPa})$ & $\eta(\mathrm{GPa} \cdot \mu \mathrm{s})$ \\
\hline 8.92 & 0.09 & 0.26 & 0.23 & 0.1 \\
\hline
\end{tabular}




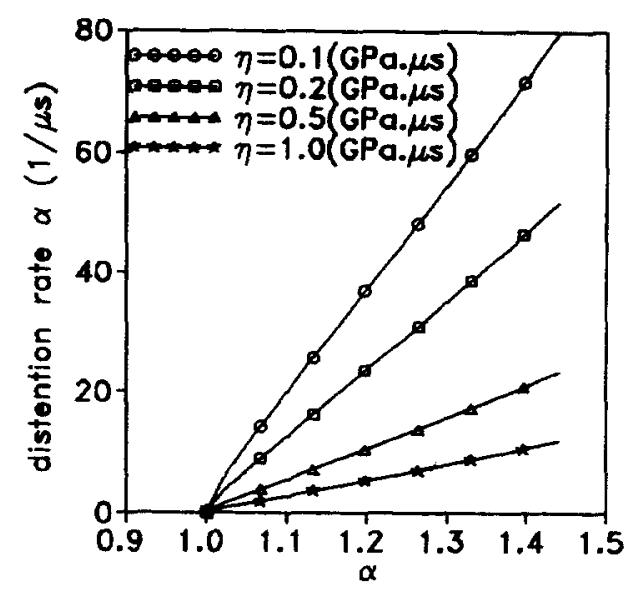

(a)

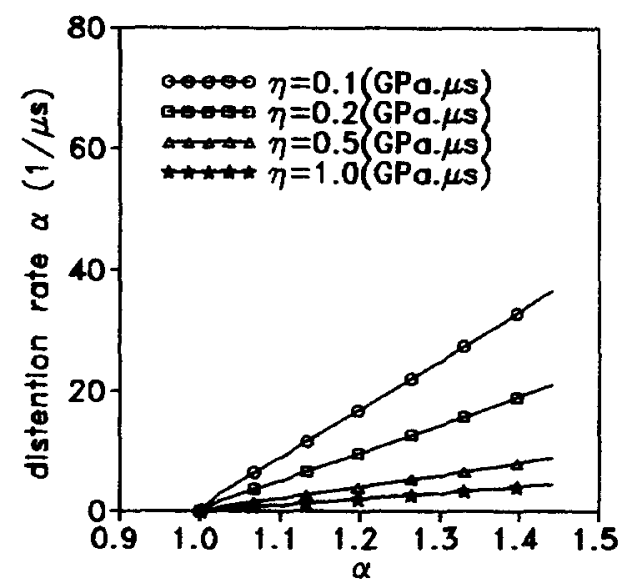

(b)

Fig. 2. Influence of material viscosity $\eta$ with different values on the increment of distention rate $\dot{\alpha}$ under different loading rates. (a) $G=0.3$, (b) $G=0.1$.

the material viscosity plays an important role in the behavior of dynamic ductile damage of the solids, especially for the high loading rates $\left(\dot{\epsilon} \geqslant 10^{3} \mathrm{~s}^{-1}\right)$.

The inertial effects are investigated through the numerical analysis of the evolution eq. (30), in which the influence of inertia is included, and eq. (31), in which the influence of inertia is neglected. The results of the comparison of the void growth obeying eqs (30) and (31) with the different external loading rates are shown in Fig. 3. The profiles in Fig. 3 indicate clearly that the inertial effects on the dynamic growth of voids are significant. The smaller the material viscosity is [namely, the higher the strain rates are due to eq. (37)], the greater the inertial effects are. It also shows that the inertial effects become much greater as the distention $\alpha$ increases. Moreover, an important conclusion is obtained that when the strain rate $\dot{\epsilon} \geqslant 10^{3} \mathrm{~s}^{-1}$, the inertial effects may almost be neglected, i.e. the influence of inertia is a special phenomenon under the case of intense dynamic loading.

The coalescence of voids is found to have two modes, i.e. direct impingement and concerned by microcracks which are composed of much smaller microvoids. The behavior of coalescence of voids is extremely complex. In general, it is assumed that coalescence takes place when distention $\alpha$ reaches some value $\alpha_{\text {crit, }}$, called critical distention which can be determined by 


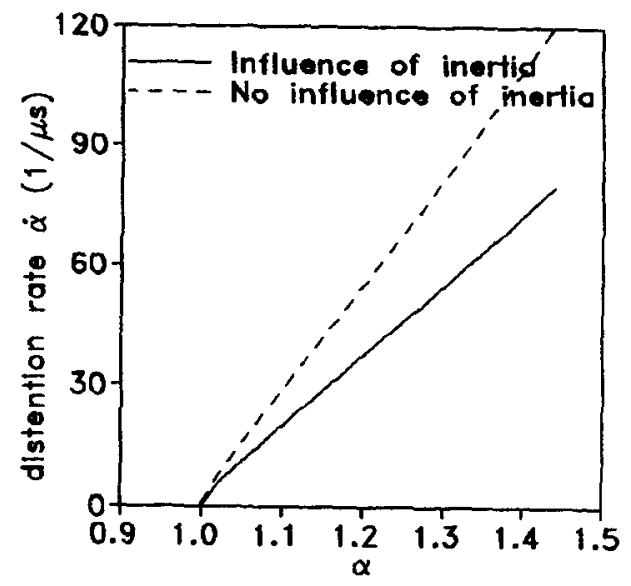

(a)

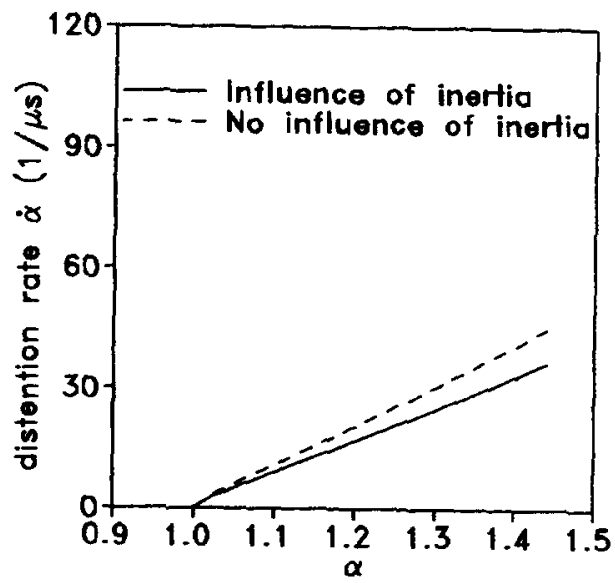

(b)

Fig. 3. Influence of inertia on the increment of distention rate $\dot{\alpha}$ under different loadings. (a) $G=0.3$, (b) $G=0.1 ;$ for both (a) and (b), $\eta=0.1(\mathrm{GPa} \cdot \mu \mathrm{s})$.

experimental measurement. For pure copper, $\alpha_{\text {crit }} \approx 1.43[12]$. The calculation stops when $\alpha \geqslant \alpha_{\text {crit. }}$

\section{NUMERICAL CALCULATIONS}

\subsection{Plate-impact test}

As an application of the foregoing theory, a spallation experiment on LY12 aluminum alloy was simulated. It is a plate-impact experiment in which a $5.28 \mathrm{~mm}$-thick LY12 aluminum alloy plate strikes a $9.92 \mathrm{~mm}$-thick LY12 aluminum alloy target. Specimens were machined into circular plates $70 \mathrm{~mm}$ in diameter. The experiment was performed with a $101 \mathrm{~mm}$ bore single-stage light gas gun. The schematic arrangement for the experiment is shown in Fig. 4. The impact velocity is $0.35 \mathrm{~mm} / \mu \mathrm{s}$. The specimen was soft recovered with a specially designed catcher to prevent any secondary damage. The recovered specimen was finally sectioned along the diameter into two parts for measuring the distribution of postimpact porosity along the symmetric axis of the spalled LY12 aluminum alloy sample by means of quantitative metallographic examination. Measured pressure-time history is shown in Fig. 5. 


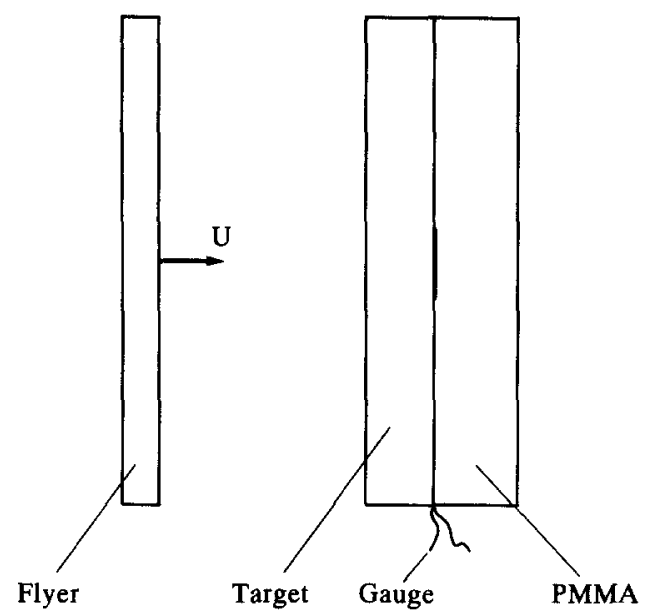

Fig. 4. Plate-impact apparatus for spall study.

\subsection{One-dimensional finite-difference calculation}

The one-dimensional flow equations in terms of the Lagrangian position coordinate $x$ are

$$
\left\{\begin{array}{l}
\frac{\partial \epsilon}{\partial t}+\frac{\partial u}{\partial x}=0 \\
\rho_{0} \frac{\partial u}{\partial t}-\frac{\partial \sigma}{\partial x}=0 \\
\rho_{0} \frac{\partial E}{\partial t}-\sigma \frac{\partial u}{\partial x}=0
\end{array}\right.
$$

where

$$
\epsilon \equiv 1-\frac{\rho_{0}}{\rho},
$$

$t$ is the time, $\rho$ is the density ( $\rho_{0}$ is the initial density), $u$ is the particle velocity in the $x$ direction, $\sigma$ is the longitudinal stress component and $E$ is the internal energy per unit mass.

The material constitutive equation is written in terms of the macroscopic mean stress (pressure) $P$ and the deviatoric stress components $S_{i j}$. In the model described here, void growth is only related to the mean stress $P$. The deviatoric stress components depend on the shear modulus $\mu$ and yield strength $Y$, which are both functions of the porosity $\Phi$. The plastic yield condition for the solid is

$$
\frac{3}{2} S_{i j} S_{i j} \leqslant Y^{2} .
$$

No attempt is made to include work-hardening and rate-dependent terms of the type used in eq. (4) for calculations of the wave profiles. These effects are still poorly understood themselves and do not greatly influence the fracture process. This treatment can simplify the calculation greatly.

In the elastic region $\left(3 S_{i j} S_{i j}<2 Y^{2}\right)$ the stress deviatoric rates are given by

$$
\frac{\mathrm{d} S_{i j}}{\mathrm{~d} t}=2 \mu\left(\frac{\mathrm{d} \epsilon_{i j}}{\mathrm{~d} t}-\frac{1}{3} \delta_{i j} \frac{\mathrm{d} \epsilon}{\mathrm{d} t}\right)
$$

It is assumed that the function which relates pressure specific volume and specific internal energy for the matrix material in the porous state is the same as that which relates these quantities for the matrix material in its nonporous state. With this assumption, the $P-\Phi$ model gives the pressure in the porous material as a function of specific volume, specific internal energy and porosity. The form of this function is determined by the pressure-specific volume specific internal energy (PVE) function 


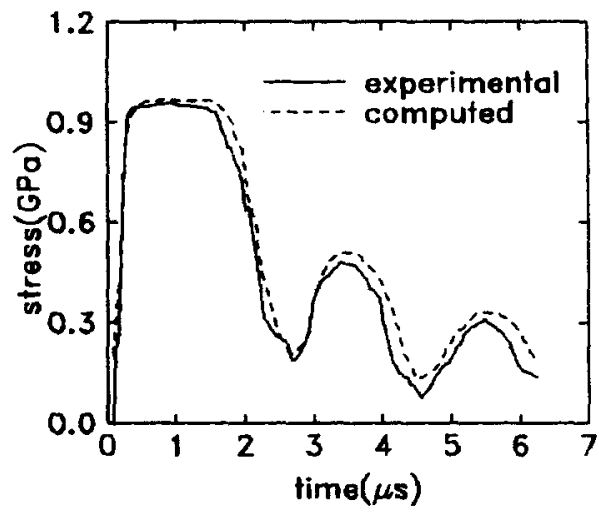

Fig. 6. Comparison of stress record from manganin gage in PMMA behind LY12 aluminum alloy with computed stress.

for the matrix material in its nonporous state. We use the following PVE relation for the porous material [10]

$$
P=(1-\Phi) P_{\mathrm{s}}\{(1-\Phi) V, E\},
$$

where $V$ is the specific volume for the porous material which is defined as $V=\rho_{0} / \rho$, the specific internal energy for the matrix material is the same in the porous and nonporous conditions, and the specific internal energy of the porous material is that of the matrix material, i.e. the surface energy of the pores is neglected. The PVE relation of the matrix material is given by [10]

$$
P_{\mathrm{s}}\{(1-\Phi) V, E\}=K_{0}\left[\frac{1}{(1-\Phi) V}-1\right]+\Gamma \rho_{\mathrm{s}} E,
$$

where $K_{0}$ is the adiabatic bulk modulus at zero pressure, $\Gamma$ is the Gruneisen coefficient and here $\rho_{\mathrm{S}} \Gamma$ is assumed to be a constant given by its low-pressure value $\rho_{0} \Gamma_{0}$.

The relation between the macroscopic yield strength $Y$ and porosity $\Phi$ is given by [10]

$$
Y=(1-\Phi) Y_{0} .
$$

The shear modulus is assumed to be degraded by the presence of voids. The relation between the shear modulus $\mu$ and porosity $\Phi$ is suggested by Mackenzie [14]

$$
\mu=\mu_{\mathrm{S}}(1-\Phi)\left(1-\frac{6 K_{0}+12 \mu_{\mathrm{S}}}{9 K_{0}+8 \mu_{\mathrm{S}}}+\Phi\right)
$$

where $\mu_{\mathrm{S}}$ is the material shear modulus in the solid surrounding the void.

The relation between $\Phi_{\mathrm{g}}$ and $\dot{\alpha}$ is given by

$$
\Phi_{\mathrm{g}}=\dot{\alpha} / \alpha^{2} .
$$

Table 2. Material parameters for spallation calculations

\begin{tabular}{lcc}
\hline & Aluminum & PMMA $\dagger$ \\
\hline$\rho_{0}\left(\mathrm{~g} / \mathrm{cm}^{3}\right)$ & 2.79 & 1.185 \\
$K_{0}(\mathrm{GPa})$ & 86.2 & 8.13 \\
$\Gamma_{0}$ & 1.7 & \\
$Y_{0}(\mathrm{GPa})$ & 0.33 & \\
$\mu_{\mathrm{S}}(\mathrm{GPa})$ & 37.5 & \\
$\eta(\mathrm{GPa} \cdot \mu \mathrm{s})$ & 0.001 & \\
$\sigma_{\mathrm{n}}(\mathrm{GPa})$ & 1.7 & \\
$\Phi_{\text {crit }}$ & 0.28 & \\
\hline
\end{tabular}

†PMMA is treated in these calculations as a fluid (no shear strength). 


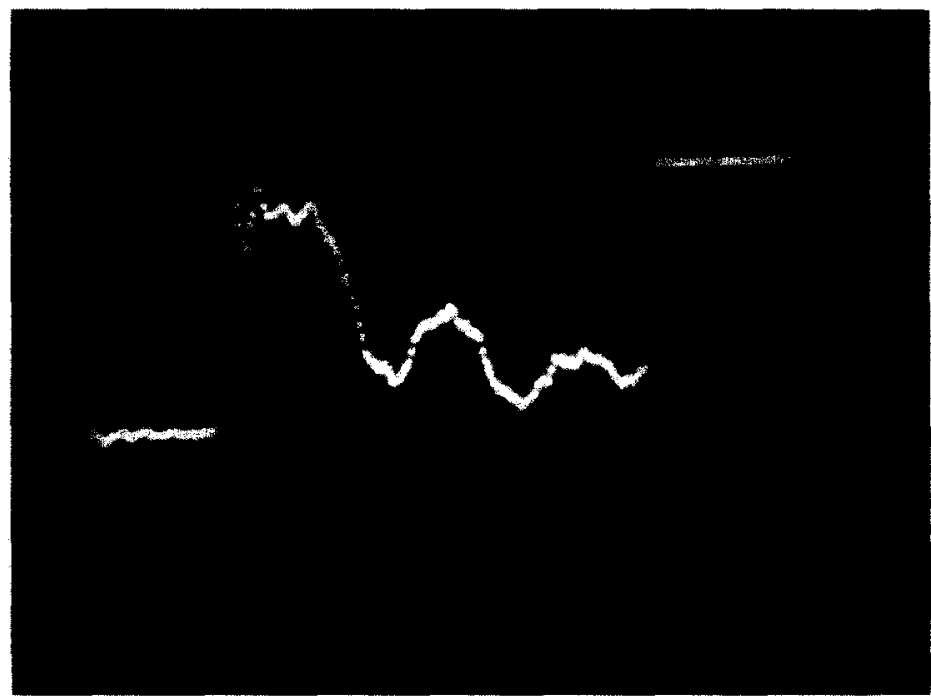

Fig. 5. Stress record from manganin gage in PMMA behind LY12 aluminum alloy specimen. 


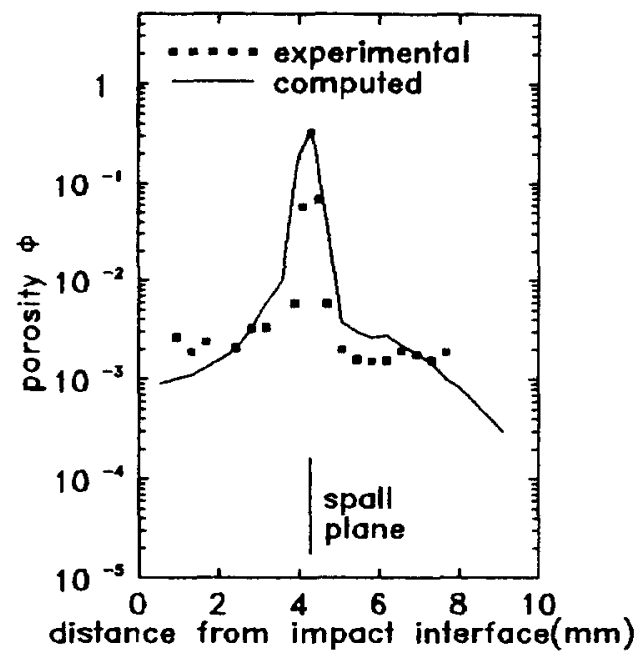

Fig. 7. Comparison of calculated (solid line) and measured (data points) postimpact porosity in LY 12 aluminum alloy sample.

An application of the dynamic failure analysis to the problem of time-dependent spallation in LY12 aluminum alloy gives a very good representation of the data (Fig. 6), with the material parameters listed in Table 2. Comparison of the calculated final porosity, or void volume, with the experimental measurement is shown in Fig. 7. The computed distribution is reasonably good.

\section{SUMMARY}

A dynamic ductile failure model is developed in which the nucleation and growth of voids and, especially, inertial effects are taken into account. The closed form of the evolution equation of voids is obtained. Numerical calculations show that the inertial effects appear to resist the growth of voids. The higher the strain rates are, the greater the inertial effects are. It is suggested that the inertial effects should not be neglected in the whole process of spall fracture. It is also indicated that the dynamic growth of voids is highly sensitive to the strain rates. Spall fracture of LY12 aluminum alloy for plate-impact conditions is successfully described with the model.

Note: LY12 aluminum alloy is the same as 2024 aluminum alloy.

\section{REFERENCES}

[1] M. A. Meyers and C. T. Aimone, Prog. Mater. Sci. 28, 1-96(1983).

[2] D. E. Grady, J. appl. Phys. 53, 322-325 (1982).

[3] G. Regazzoni, J. N. Johnson and P. S. Follansbee, J. appl. Mech. 53, 519-528 (1986).

[4] V. Tvergaard and A. Needleman, J. Mech. Phys. Solids 34, 213-241 (1986).

[5] J. N. Johnson, J. appl. Phys. 52, 2812-2825 (1981).

[6] D. R. Curran, L. Seaman and D. A. Shockey, Physics Reports 147, 254-388 (1987)

[7] C. C. Chu and A. Needleman, J. Engng Mater. Technol. 12, 249-256 (1980).

[8] M. M. Carroll and A. C. Holt, J. appl. Phys. 43, 1626-1636 (1972).

[9] W. Johnson and P. B. Meller, Engineering Plasticity. Van Nostrand Reinhold, London (1973).

[10] L. Seaman, D. R. Curran and D. A. Shockey, J. appl. Phys. 47, 4814-4826 (1976).

[11] R. Cortes, Int. J. Solids Structures 29, 1339-1350 (1992).

[12] Z. P. Wang, Acta Mech. Sol. Sinica 6, 69-80 (1993).

[13] L. C. Chhabildas and J. R. Say, J. appl. Phys. 50, 2749-2756 (1979).

[14] J.H. Mackenzie, Proc. Phys. Soc. 63B, 2-11 (1950).

[15] V. K. Luk, M. J. Forrestal and D. E. Amos, J. appl. Mech. 58, 1-6 (1991). 